\title{
Design on Dance Appreciation Video System Based on MVC Mode
}

\author{
Xiaojie Wang \\ College of Art and Communication, Bohai University, Jinzhou, P.R. China \\ 1019813588@qq.com
}

Keywords: MVC mode; dance appreciation; video system; structure design

\begin{abstract}
Dance appreciation is a creative and complex spiritual activity process of the unity of feeling and understanding, affection and understanding. Development of information technology and mobile terminals provide a new way to dance appreciation. In order to develop dance appreciation video system, this paper designed it based on the MVC mode and provided technical support for software development. Firstly, studied the MVC mode, using graph represented the relationship among modes, views and controllers; and then designed layered system architecture consisting of user layer, service layer and management layer; finally, with the live video of one of the core program designed the program flow and illustrated the implementation process. The results shown that forcibly defining controller, model, view and other modules of application improved the readability of application and the reusability of code.
\end{abstract}

\section{Introduction}

Dance is a beautiful art, beauty and fun in which. Dance is the art of love, affection and meaning in which. Dance derived from the life and higher than the life, after refining, organizing and beautify the human action art, is a kind of comprehensive dynamic selection art with music, lights, set, particular garment. Dance appreciation is a creative and complex spiritual activity process of the unity of feeling and understanding, affection and understanding. Need to experience, understand, associate, image positive mental activities and analysis, integrate image thinking in order to achieve concrete grasp for dance works, and then to complete a relatively perfect dance aesthetic process $[1,2]$. Due to space constraints, dance appreciation has always been the aristocratic luxury in a long time. The development of information technology and mobile terminal provide technical assurance for video system, computer image processing capabilities and network communication technology continues to mature, the video system enter a rapid development period, which provide a new way to dance appreciation. The majority of dance lovers may from time and space constraints, through the network video for dance appreciation. In order to develop dance appreciation video system, this paper designed it based on MVC mode and provided technical support for software development.

\section{MVC Design Mode Structure}

Frame technology is development model based on applications in certain areas, provides a large number of software reuse, provides developers with a unified software development kits and mode architecture, makes software developers escaped from the tedious preparation of the code, and focuses on researching business of logic application and shielding the underlying implementation details of the code, thereby improving development efficiency. In order to meet the needs of different applications can improve and expand the function of framework on the basis of application and analyzing. MVC (Model-View-Controller), that is the first letter of the model, view and controller, makes the application input and business logic output separated according to the Model, View, and Controller, an application is divided into the model layer, view layer and control layer $[3,4]$. The structure is shown in Fig.1.

Model is a part of processing the application data logic in application; usually the model object is responsible for access to data in the database. Business logic processes is black-box operation for other layers, the model accepts the requested data of view, and returns the final results; the view is a showing part of processing data in the application, that is the interface of user and application, 
usually view is created based model data. User or developer does not need to master database knowledge and logic operations; the controller is part of processing user interaction in the application, controller is usually responsible for reading data from view, controlling user input and transmitting the data to model [5-7].

The separation of model, view and controller makes a model can have multiple views; a model can be reused for multiple views, improved code reusability. Model is relatively independent, to modify business logic without having to involve controllers and views, easy to expand, maintain and transplant the program. If the user used a view of the controller changing the data of model, all other views that depend on these data should reflect these changes. Therefore, regardless of what happened when the data changes, the controller will notify all of the view, which is actually a model change propagation mechanism.

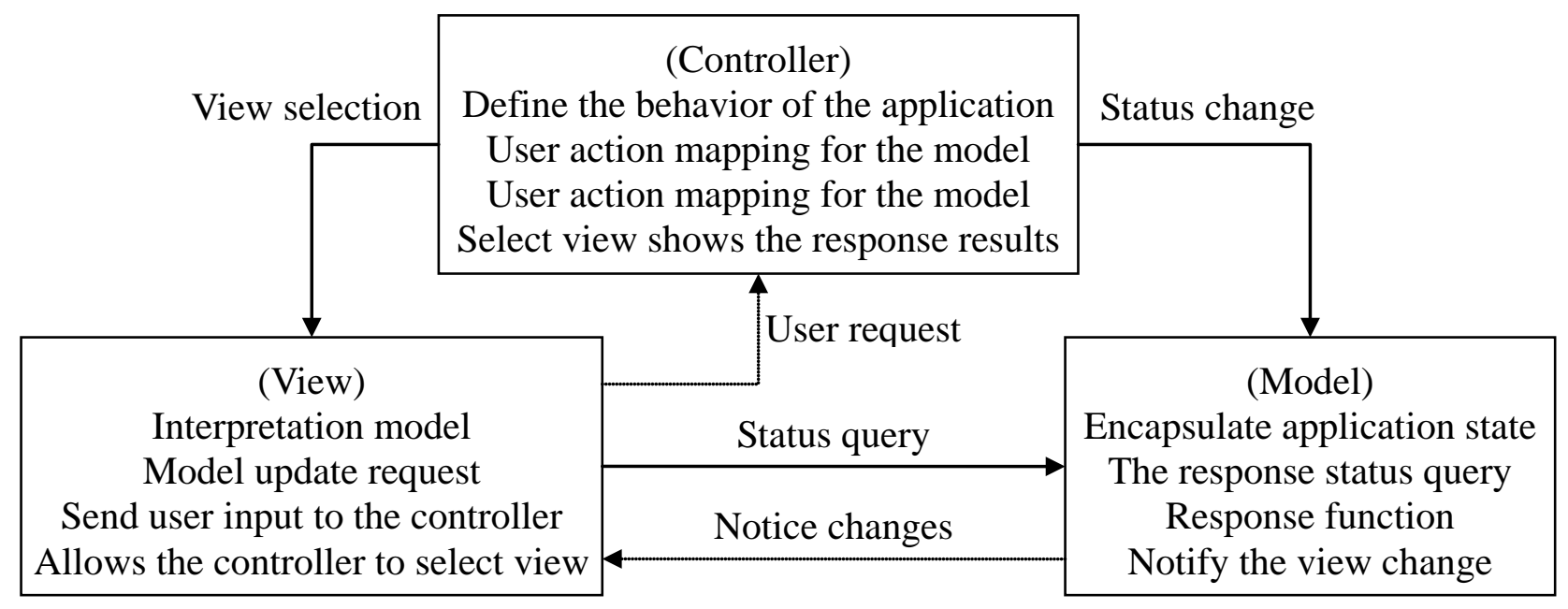

Fig. 1. MVC design mode structure

\section{System Structure Design}

Dance appreciation video system consists of the user layer, service layer and management layer, system structure is shown in Fig. 2.

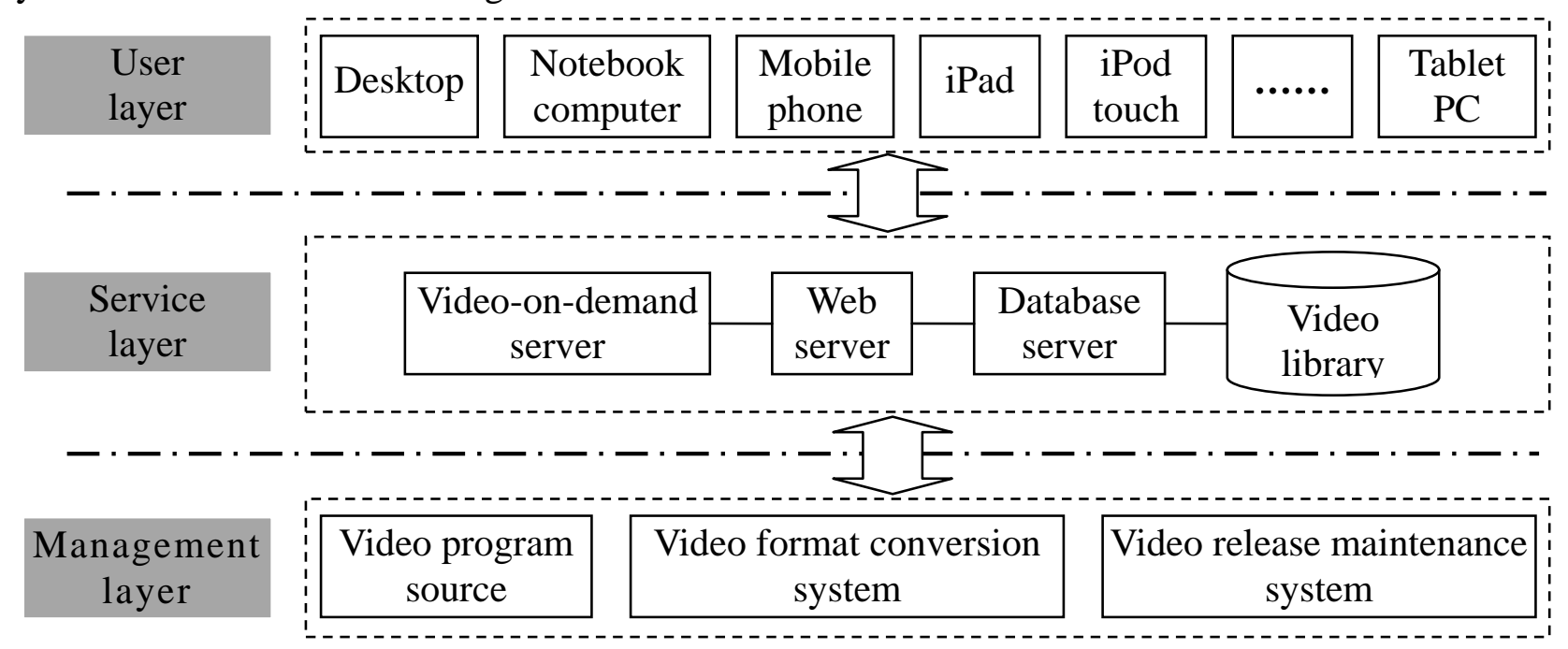

Fig. 2. System structure of dance appreciation

User layer is mainly terminal equipment used by the user, including Desktop, Notebook computer, Mobile phone, iPad, iPod touch, Tablet PC and so on. Network connection can use LAN, WAN, Internet, WiFi, 3G, etc.

Service layer is the core layer of the system, mainly composed of the WEB server, database server, video-on-demand, video library and other modules. WEB server is used to store program 
files; data base server is used to store basal data and user data; video-on-demand server is used to broadcast video; video library is used to store all video files.

Management layer for administrators operating, administrator privileges can be obtained through the system. Management layer consists of video release maintenance system, video format conversion system and video program source. Through management layer achieved uploading video files and category management, as well as adding, modifying, deleting and other operations for files.

\section{Core Programming}

The core program of dance appreciation video system is live video and video on demand, take live video for example, the program design process is shown in Fig. 3 [8].

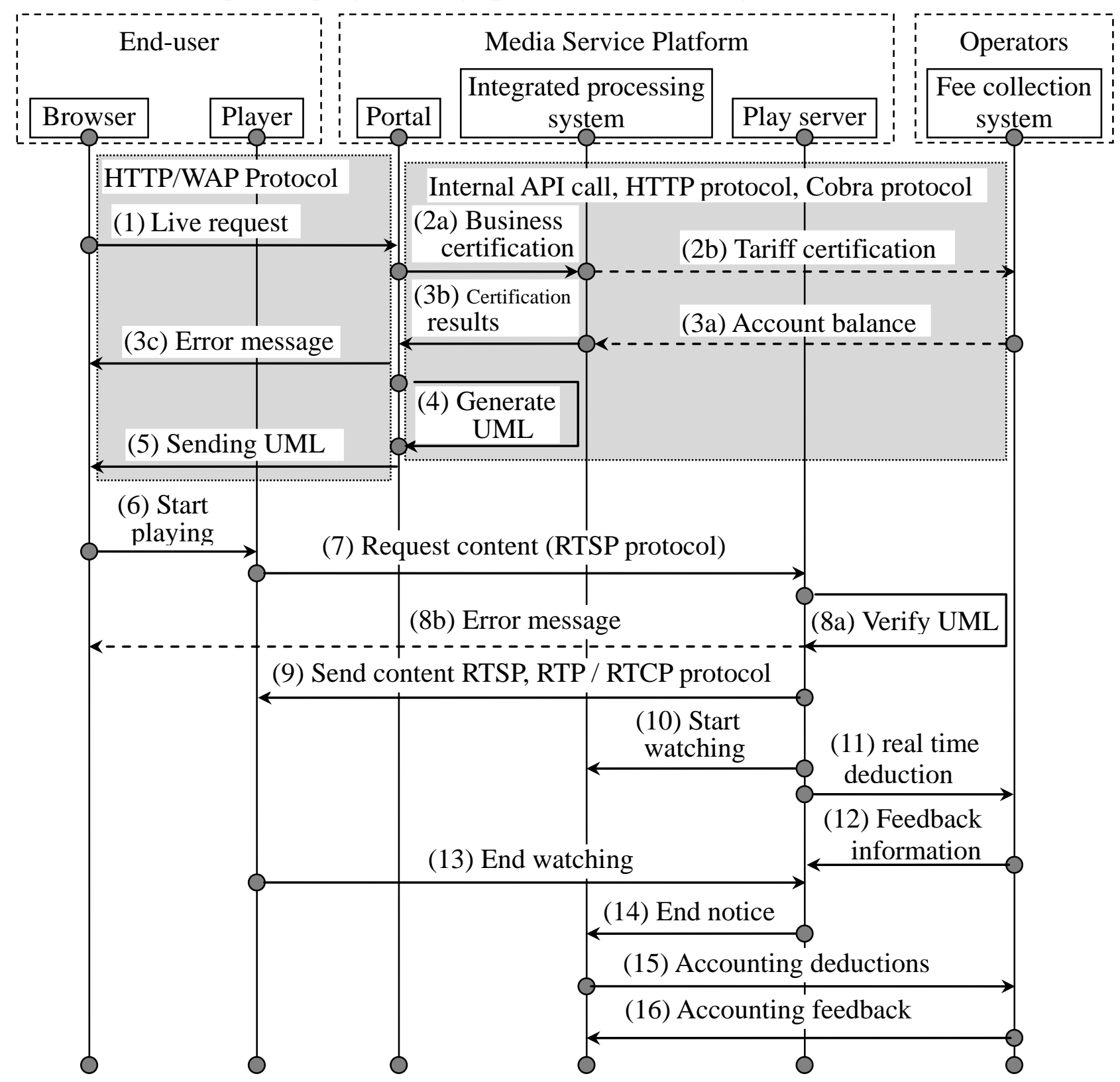

Fig. 3. Programming process on live video

Description of the live system program flow diagram shown in Fig. 3 as follows: End user issued a live video request to portal outlets; Portal carries on business certification for the user and access tariff information; Including account balances, the certification result and error message; Portals make video content address generate new UML after obtained additional validation code; Portal sent UML with a verification code to the mobile end-users; After the mobile terminal received the 
URL information with the validation code automatically start streaming media player; Play server requests streaming content under target URL; Verification is right to next step; otherwise, it displays an error message and returns; Play server sends streaming content to the mobile terminal; Play server sends notifications to the integrated processing system and prompts the user to start watching; Charge a fee, if the balance is sufficient to continue to watch, otherwise stops watching; Returns the user's deductions and query information; User end watching voluntarily or terminate for other reasons; Play server sends the time, reasons and other information of ending view for integrated processing system; Integrated treatment system generates charge information, charge system obtains charge; Charge system returns to the charging information.

\section{Conclusion}

Dance is a form of artistic expression with unique beauty in the art aesthetic, either can pass emotion, or can obtain beauty, it integrated the time and space, shown harmonious rhythm, not only beautify the art of human action, but also beyond the appreciation of beauty about body and soul. The dance appreciation video system developed in this study can achieve live video and video on demand; also can play as a television program. This paper based on the MVC mode, forcibly defining controller, model, view and other modules of application improved the readability of application and the reusability of code [9].

\section{Acknowledgement}

This work is supported by social science fund project of Liaoning province (L12DWJ018).

\section{References}

[1] Q. Q. Wang, "The relationship between dance and aesthetic appreciation," Art and Literature for the Masses, vol. 58, no. 7, pp. 179, 2013.

[2]

Baidu libary,

"Dancing

appreciation,"

http://wenku.baidu.com/link?url=SbmYG8vEaJJzge2NekjI1u33wvB39xMV27HEu5EPOHDex

E9AU4BAEjr3u7XZdiEVwKJXRpbBEUwoj_gqS1-7dc3IIEvAZ96Pdgsd6YoTmG7, 2015-6-8.

[3] M. Xu, "The Triple Master Business Management System Design and Implement Based On the SSH2 Framework of MVC Mode," Master's degree of Ocean University of China, 2010.

[4] Dragos-Paul Pop, Adam Altar, "Designing an MVC Model for Rapid Web Application Development," Procedia Engineering, vol. 69, no. 1, pp. 1172-1179, 2014.

[5] Ying Chen, Miska M. Hannuksela, Teruhiko Suzuki, Shinobu Hattori, "Overview of the MVC + D 3D video coding standard," Journal of Visual Communication and Image Representation, vol. 25, no. 4, pp. 679-688, 2014.

[6] Rong-Hua Li, Jeffrey Xu Yu, Xin Huang, et al, "Measuring the impact of MVC attack in large complex networks," Information Sciences, vol. 278, no. 10, pp. 685-702, 2014.

[7] Xuan Yin, Wei Zheng, Ming Zhang, et al, "A modularized operator interface framework for Tokamak based on MVC design pattern," Fusion Engineering and Design, vol. 89, no. 5, pp. 628-632, 2014.

[8] G. M. Lu, "Mobile Streaming Media Technology," Electronics Industry Press, 2010.

[9] C. Goktug Gurler, Anil Aksay, Gozde Bozdagi Akar, A. Murat Tekalp, "Architectures for multi-threaded MVC-compliant multi-view video decoding and benchmark tests," Signal Processing: Image Communication, vol. 25, no. 5, pp. 325-334, 2010. 